\title{
A resonant instability of steady mountain waves
}

\author{
By YOUNGSUK LEE, ${ }^{1}$ DAVID J. MURAKI ${ }^{1}$ \\ AND DAVID E. ALEXANDER ${ }^{2}$ \\ ${ }^{1}$ Department of Mathematics, Simon Fraser University, Burnaby, BC V5A 1S6, Canada \\ ${ }^{2} 3$-Sigma Consulting, North Vancouver, BC V7L 3G3, Canada
}

(Received 23 November 2005 and in revised form 17 May 2006)

\begin{abstract}
A new mechanism for the instability of steady mountain waves is found through analysis of the linear stability problem. Steady flow of a hydrostatic stratified fluid is known to be unstable when the streamlines are at, or very close to, overturning. When the topography has multiple peaks, it is shown that this criterion can be superseded by an instability owing to a resonant triad interaction. For flow over two peaks, the threshold heights for instability are roughly half those which produce overturning streamlines. The mechanism behind the instability is the parametric amplification of counter-propagating gravity waves. The resonant nature of the instability is further illustrated by the existence of discrete peak-to-peak separation distances where the growth rate is a maximum.
\end{abstract}

\section{Introduction}

A mountain, or lee wave is a form of internal gravity wave generated by the flow of stably stratified air over mountainous terrain (see the general review by Baines 1995). These waves, often visualized by downstream cloud patterns, are implicated in severe lee-side downslope windstorms and clear air turbulence. Propagation of these waves is important to the transport of momentum and energy in the atmosphere (Smith 1979). The breaking of gravity waves is also a common initiator for cascades to small-scale turbulence (Wurtele, Sharman \& Datta 1996, and references therein). As the instability analysed here provides a dynamical means to produce flow overturning, it represents yet another efficient pathway for the generation of small scales.

The nonlinear theory of mountain waves begins with the discovery by Long (1953) that a steady theory for stratified flow over two-dimensional topography can be exactly expressed as a single equation for streamfunction. Furthermore, in the special case of linear stratification and uniform upstream wind, the nonlinear steady streamfunction satisfies a linear Helmholtz equation. The wave solutions of Long's theory are wellunderstood from analytical descriptions (Miles \& Huppert 1968) and numerical solutions (Lilly \& Klemp 1979; Laprise \& Peltier 1989c; Muraki 2005). These steady flows have been observed to be stable in both laboratory experiments (Long 1955; Baines \& Hoinka 1985), and numerical computations (Clark \& Peltier 1977; Klemp \& Lilly 1978).

A counterpoint to this theory of stable steady waves over topography is the wellestablished fact that periodic plane gravity waves are subject to a nonlinear instability. Floquet theory (Mied 1976; Drazin 1977; Klostermeyer 1982) has shown that a sinusoidal gravity wave, even of infinitesimal amplitude, is parametrically unstable through resonant wave interactions (see also the review by Staquet \& Sommeria 2002). The possibility that this resonance mechanism has implications for topographic flow 
(a)

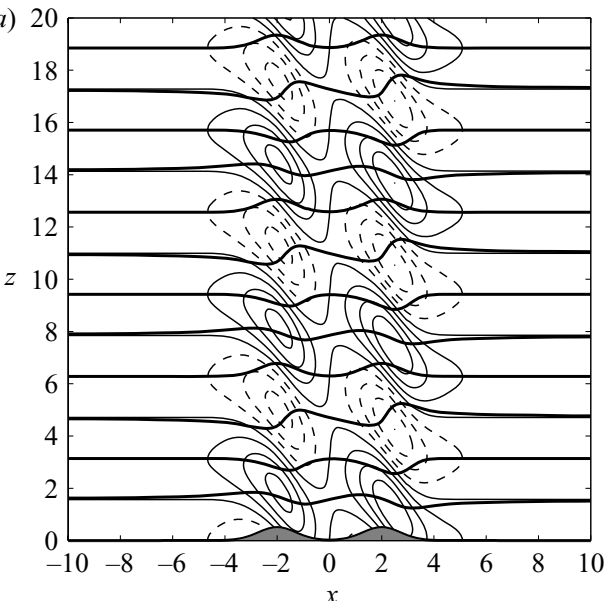

(b)

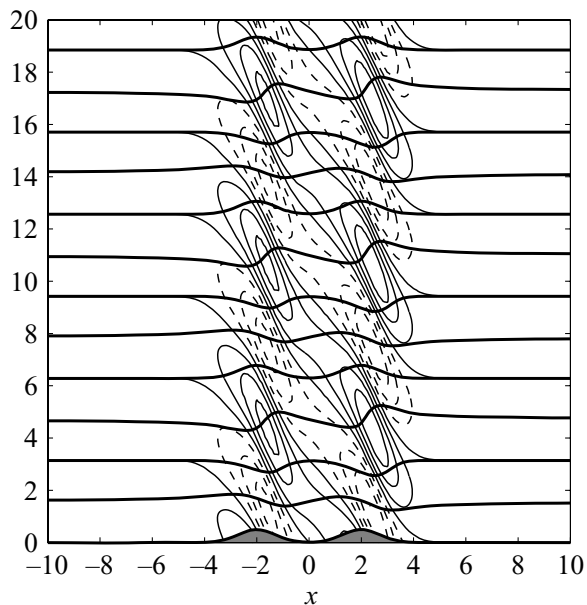

FIGURE 1. Long's steady solution for twin-peak topography with $\mathscr{D}=4$ and $\mathscr{A}=0.5$. Streamlines are shown as thick contours spaced at $\pi / 2$. The steady velocities (a) $u^{s}$ and (b) $w^{s}$ are shown as thin contours spaced at $1 / 4$ of the maximum disturbances, with negative values dashed. In $\S 3$, this flow is shown to be unstable.

was noted in the original hydrostatic simulations of Klemp \& Lilly (1978), where the theoretical steady solutions were not achieved for flow past periodic topography. The growth of small-scale instabilities, leading to the eventual destruction of the numerical solution, was attributed to resonant interactions, and not to numerical instability. This question of stability was revisited recently for non-periodic topography, where the breakdown of the steady flow via an oscillatory instability was observed in a twodimensional simulation with three (or more) isolated peaks (C. Epifanio \& D. Muraki, work in progress). In theoretical support of this finding, the present study determines that the hydrostatic steady mountain wave can indeed develop a linear instability, and that this instability clearly results from a resonant wave interaction.

Long's theory, as an exact nonlinear theory, is valid for topographic obstacles of finite height. With increasing topographic heights, a well-known consequence of the theory is the prediction of overturning streamlines. This implies the loss of static stability in localized regions of unstable stratification (Smith 1977). Under such conditions, the development of overturning flow is preceded by wavebreaking and a cascade to turbulence (Pierrehumbert \& Bacmeister 1987; Laprise \& Peltier $1989 a$ ). The nature of this wavebreaking instability at, or near, overturning conditions has received considerable attention in the literature (Pierrehumbert \& Wyman 1985; Laprise \& Peltier 1989b; Scinocca \& Peltier 1993; Afanasyev \& Peltier 1998; Eiff \& Bonneton 2000; Mayr \& Gohm 2000). Of interest here is the possibility for instability of steady flow over topography with heights well below the critical value required for streamline overturning.

Motivated by the observation of instability in simulations involving multiple-peaked topography, we formulate in $\S 2$ the linear stability problem for hydrostatic solutions of Long's theory with twin peaks (figure 1). Only steady flows without streamline overturning are considered. The linear stability problem is numerically formulated as a matrix eigenvalue problem. The typical matrix dimension is of the order $10^{5}$, so a sparse-matrix algorithm is used to identify unstable eigenvalues. In stability calculations for topographies with four values of peak separation, each is found to 


$\begin{array}{ccccccc}\text { Variable } & x & z & u & w & \theta & \phi \\ \text { Scale } & \mathscr{L} & \mathscr{U} / \mathscr{N} & \mathscr{U} & \mathscr{U}^{2} / \mathscr{N} \mathscr{L} & \mathscr{U} \mathscr{N} \theta_{r} / g & \mathscr{U}^{2}\end{array}$

TABLE 1. The dimensional scalings for the non-dimensionalization. The variables and scales are defined in $\S 2.1$.

have several unstable eigenvalues (§3). Spatially, the most unstable modes appear to have a complicated wave structure, but in fact, are primarily comprised of three distinct linear-like wavepackets. Two of these wavepackets have counter-propagating group velocities, and are found to form a resonant triad with the steady flow (§4). The mechanism for instability is a parametric amplification of these wavepackets within a localized region above the topography. For clearly separated obstacles, the onset of oscillatory instabilities is found to occur when the heights are roughly half that required for critical overturning ( $(5)$. Analogous with the behaviour of a resonant cavity, the computed unstable modes are found to be organized into a hierarchy of branches, with subsequent branches having spatial structures with increasing cellularity $(\S 6)$. Finally in $\S 7$, we interpret this topographic instability as a localized variation of the resonant instability of the periodic plane wave.

\section{Long's steady flow and its linear stability theory}

\subsection{The primitive equations for topographic flow}

The governing equations are taken to be the incompressible Euler equations under the Boussinesq and hydrostatic approximations. The background state of the atmosphere is idealized to a uniform upstream horizontal wind and a linear stratification of potential temperature in the vertical. Relative to this background, the non-dimensional primitive equations for disturbances are

$$
\left.\begin{array}{rl}
u_{x}+w_{z} & =0, \\
\frac{\mathrm{D} u}{\mathrm{D} t} & =-\phi_{x}, \\
-\theta & =-\phi_{z}, \\
\frac{\mathrm{D} \theta}{\mathrm{D} t}+w & =0,
\end{array}\right\}
$$

where $u, w$ are the horizontal and vertical velocities, $\theta$ is the potential temperature, and $\phi$ is the pressure, or geopotential height (Salmon 1998). The advective derivative, including the total horizontal wind $u^{T}=1+u$ is now

$$
\frac{\mathrm{D}}{\mathrm{D} t}=\frac{\partial}{\partial t}+u^{T} \frac{\partial}{\partial x}+w \frac{\partial}{\partial z}
$$

and the total stratified potential temperature is $\theta^{T}=z+\theta$. The absence of the vertical acceleration term $\mathrm{D} w / \mathrm{D} t$ in the third equation of (2.1) is a consequence of the hydrostatic assumption.

The dimensional scalings which result in (2.1) are summarized in table 1 . The background state is characterized by the upstream wind $\mathscr{U}$, and the linear stratification (Brunt-Väisälä frequency) $\mathscr{N}$. The simplest characterization of topography involves only the mountain width scale $\mathscr{L}$, and the height $\mathscr{H}$. The factor $\theta_{r} / g$, where $\theta_{r}$ is a 
reference potential temperature and $g$ is the gravitational constant, converts buoyancy acceleration to potential temperature.

The flow domain is the semi-infinite $(x, z)$-plane, unbounded above $(z \rightarrow \infty)$ and bounded below by the non-dimensional topographic surface

$$
z=\mathscr{A} h(x)
$$

where $\mathscr{A}=\mathscr{N} \mathscr{H} / \mathscr{U}$ is a dimensionless topographic height based on the natural vertical scale $\mathscr{U} / \mathscr{N}$ of the hydrostatic gravity waves. For the twin-peak topography, we have chosen the $O(1)$ surface profile to be the sum of two Gaussians separated by a peak-to-peak distance $\mathscr{D}$

$$
h(x)=\exp \left(-(x+\mathscr{D} / 2)^{2}\right)+\exp \left(-(x-\mathscr{D} / 2)^{2}\right) .
$$

This minimal characterization of twin-peak topography through a height $\mathscr{A}$ and peak separation $\mathscr{D}$ is the basis for the parameter studies found in $\S \S 5$ and 6.1 .

Two-dimensional incompressibility allows for the formulation in terms of a streamfunction $\psi(x, z, t)$ with $u=\psi_{z}$ and $w=-\psi_{x}$. The governing equations (2.1) can then be rewritten in the vorticity/buoyancy formulation (Phillips 1968; Laprise \& Peltier 1989a)

$$
\left.\begin{array}{r}
\psi_{z z t}+\psi_{x z z}+\theta_{x}+J\left(\psi_{z z}, \psi\right)=0 \\
\theta_{t}+\theta_{x}-\psi_{x}+J(\theta, \psi)=0
\end{array}\right\}
$$

where $J(f, \psi)=f_{x} \psi_{z}-f_{z} \psi_{x}=\left(u \partial_{x}+w \partial_{z}\right) f$ is the Jacobian determinant. In the hydrostatic limit, the cross-stream vorticity is reduced to $u_{z}=\psi_{z z}$. Streamlines of the flow are identified by contours of the total streamfunction, $\psi^{T}=z+\psi$.

We impose appropriate boundary conditions for $\psi$ and $\theta$. It is assumed that there are no disturbances far upstream, hence

$$
\psi(-\infty, z, t)=0, \quad \theta(-\infty, z, t)=0,
$$

and $h(-\infty)=0$. As the topographic surface must always be a streamline of the flow, setting the total streamfunction $\psi^{T}$ to zero on the topographic surface gives the first of two lower boundary conditions

$$
\mathscr{A} h(x)+\psi(x, \mathscr{A} h(x), t)=0, \quad \mathscr{A} h(x)+\theta(x, \mathscr{A} h(x), t)=0 .
$$

For purposes of the stability analysis, the second condition restricts the total potential temperature $\theta^{T}$ on the surface to remain fixed at the upstream quiescent value.

To model an atmosphere that is unbounded above we impose a radiation condition, which ensures that disturbances are either decaying in the vertical, or are waves carrying energy upward, away from the topographic obstacle. In the stability computation, this is implemented through the introduction of a damping layer at the top of the numerical domain.

\subsection{Linear gravity waves}

In the linear limit, and neglecting boundaries, the primitive equations (2.5) admit wave solutions for each Fourier wavevector $\boldsymbol{k}=(k, m)$. These are the internal gravity waves and have the form

$$
\left[\begin{array}{l}
\psi \\
\theta
\end{array}\right]=\alpha\left[\begin{array}{c}
1 \\
\mp|m|
\end{array}\right] \exp \left(\mathrm{i}\left(\boldsymbol{k} \cdot \boldsymbol{x}-\omega_{\bar{\mp}}(\boldsymbol{k}) t\right)\right),
$$


where the amplitude $\alpha$ is an arbitrary constant (Phillips 1968). The dispersion relation for the frequency $\omega_{\mp}(\boldsymbol{k})$ is

$$
\omega_{\mp}(\boldsymbol{k})=k\left(1 \mp \frac{1}{|m|}\right),
$$

so that the horizontal phase velocities satisfy the inequality

$$
\frac{\omega_{-}(\boldsymbol{k})}{k}<1<\frac{\omega_{+}(\boldsymbol{k})}{k}
$$

relative to the non-dimensional upstream windspeed. Thus, we refer to the linear gravity wave with $\omega_{-}(\boldsymbol{k})$ as the slow wave, and the wave with $\omega_{+}(\boldsymbol{k})$ as the fast wave. Only slow waves with vertical wavenumber $|m|=1$ are stationary in the coordinate frame. As a further aside, it is a consequence of the Jacobian nonlinearity that the Fourier mode solution (2.8) actually constitutes a nonlinear solution of (2.5).

These waves are dispersive, and their energy propagates with the group velocity, $\boldsymbol{c}_{\mp}^{g}$, as defined by the gradient of $\omega_{\mp}(\boldsymbol{k})$

$$
\boldsymbol{c}_{\mp}^{g}(\boldsymbol{k})=\left(\frac{\partial \omega_{\mp}}{\partial k}, \frac{\partial \omega_{\mp}}{\partial m}\right)=\left(1 \mp \frac{1}{|m|}, \pm \frac{k m}{|m|^{3}}\right) .
$$

Therefore, a slow gravity wave propagates energy upward when $\mathrm{km}>0$, whereas a fast gravity wave propagates energy upward when $\mathrm{km}<0$ (Baines 1995).

\subsection{Long's theory for steady flow}

Under steady conditions, the primitive equations (2.5) can be written as

$$
\begin{aligned}
\left(\theta^{s}-\psi^{s}\right)_{x}+J\left(-z+\psi_{z z}^{s}, z+\psi^{s}\right) & =0, \\
J\left(z+\theta^{s}, z+\psi^{s}\right) & =0,
\end{aligned}
$$

where $\psi^{s}(x, z)$ and $\theta^{s}(x, z)$ denote the steady solutions. Equation $(2.12 b)$ implies that the steady isentropes are exactly streamlines and therefore, based on the farupstream condition (2.6), we have $\theta^{s}=\psi^{s}$. Equation (2.12a) then implies that the steady streamfunction $\psi^{s}(x, z)$ satisfies

$$
\psi_{z z}^{s}+\psi^{s}=0
$$

and thus recovers the linear case of Long's theory (Long 1953) specific to hydrostatic flow. In addition to (2.13), the steady-flow solution must also satisfy the topographic boundary condition (2.7), and the far-field radiation condition.

For this work, steady flows were computed using the direct steady solve developed in Muraki (2005). The numerical method is spectrally accurate, and because of its non-iterative nature, computationally efficient. Figure 1 shows contours of the total streamfunction $z+\psi^{s}$, and the velocity fields $u^{s}$ and $w^{s}$ for flow over twin Gaussian peaks (2.4) with $\mathscr{D}=4$ and $\mathscr{A}=0.5$. All disturbance fields are $2 \pi$-periodic in $z$. This is consistent with the dispersion relation (2.9) since steady solutions can only be composed of slow waves with vertical wavenumber $|m|=1$. Moreover, the horizontal group velocity of these waves is zero, so all of the energy flux is directed vertically. The streamlines of figure 1 do not overturn; the critical overturning height is $\mathscr{A}_{c} \approx 0.77$ for a peak separation of $\mathscr{D}=4$. Nonetheless in $\S 3$, this flow for height $\mathscr{A}=0.5$ is shown to be linearly unstable. 


\subsection{Formulation of the stability problem}

\subsubsection{The linear stability equations}

The linear stability analysis for Long's theory introduces disturbances of the form

$$
\left.\begin{array}{c}
\psi(x, z, t)=\psi^{s}(x, z)+\operatorname{Re}\left\{\mathrm{e}^{\lambda t} \tilde{\psi}(x, z)\right\}, \\
\theta(x, z, t)=\theta^{s}(x, z)+\operatorname{Re}\left\{\mathrm{e}^{\lambda t} \tilde{\theta}(x, z)\right\} .
\end{array}\right\}
$$

The time variable can be separated via exponential factors since for the steady flow, $\psi^{s}(x, z)$ and $\theta^{s}(x, z)$ do not depend on time. Substituting (2.14) into (2.5), and using (2.13), we obtain the linear stability equations

$$
\left.\begin{array}{rl}
\lambda \tilde{\psi}_{z z}+\tilde{\psi}_{x z z}+\tilde{\theta}_{x}+J\left(\tilde{\psi}_{z z}+\tilde{\psi}, \psi^{s}\right) & =0, \\
\lambda \tilde{\theta}+\tilde{\theta}_{x}-\tilde{\psi}_{x}+J\left(\tilde{\theta}-\tilde{\psi}, \psi^{s}\right) & =0,
\end{array}\right\}
$$

by keeping only linear terms in $\tilde{\psi}$ and $\tilde{\theta}$. The form of the Jacobian, $J\left(f, \psi^{s}\right)=u^{s} f_{x}+w^{s} f_{z}$, is such that the steady velocities $u^{s}(x, z)$ and $w^{s}(x, z)$ appear as non-constant coefficients in the linear stability equations (2.15). Following (2.6) and (2.7), no disturbance conditions far upstream and zero boundary conditions at the topographic surface

$$
\begin{array}{crl}
\tilde{\psi}(-\infty, z)=0, & \tilde{\theta}(-\infty, z)=0, \\
\tilde{\psi}(x, \mathscr{A} h(x))=0, & \tilde{\theta}(x, \mathscr{A} h(x))=0,
\end{array}
$$

are imposed, in addition to the far-field radiation condition.

The linear stability equations (2.15) are a two-dimensional eigenvalue problem for the stability eigenvalue $\lambda$, with eigenfunctions $\tilde{\psi}(x, z)$ and $\tilde{\theta}(x, z)$. An eigenvalue with a positive real part indicates an unstable mode of the steady flow. The growth rate is given by $\operatorname{Re}(\lambda)>0$, and its corresponding frequency by $-\operatorname{Im}(\lambda)$.

\subsubsection{Reformulation into isentropic coordinates}

Our strategy for determining stability involves computing the eigenvalues of a matrix corresponding to a finite-difference discretization of (2.15). However, naive finite-differencing is not applicable since the topographic surface does not follow a coordinate line. We overcome this difficulty by introducing a new coordinate system

$$
\xi=x, \quad \zeta=z+\psi^{s}(x, z),
$$

where curves of constant $\zeta$ are both isentropes and streamlines of the steady flow. These coordinates are terrain-following, and the $\zeta=0$ axis corresponds to the topographic surface. The surface boundary condition $(2.16 b)$ is now simplified

$$
\tilde{\psi}(\xi, \zeta=0)=0, \quad \tilde{\theta}(\xi, \zeta=0)=0,
$$

and standard numerical discretization applies. Figure 2 shows the steady flow of figure $1(\mathscr{D}=4, \mathscr{A}=0.5)$ re-mapped into isentropic coordinates. Streamlines (thick lines) are mapped into horizontal lines since the total steady streamfunction is now the vertical coordinate. The pattern retains $2 \pi$-periodicity in the vertical. The steady velocities $u^{S}(\xi, \zeta)$ and $w^{s}(\xi, \zeta)$ are re-gridded into isentropic coordinates using cubic spline interpolation. Although the introduction of isentropic coordinates applies for topographic heights $\mathscr{A}$ right up to overturning, in practice, the resolution of the finite-differencing is limited by steepening gradients in the coefficients $u^{S}(\xi, \zeta)$ and $w^{s}(\xi, \zeta)$ 

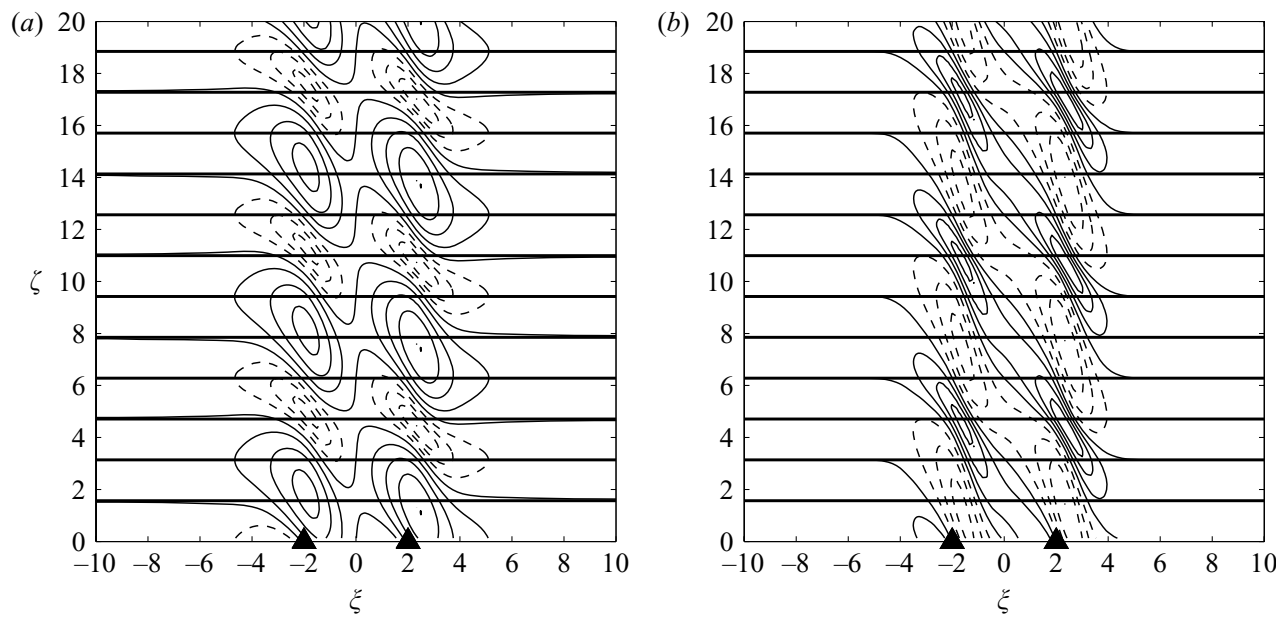

FiguRE 2. Long's steady solution of figure $1(\mathscr{D}=4, \mathscr{A}=0.5)$ remapped into isentropic $\xi, \zeta$ coordinates (2.17). Streamlines are now thick horizontal contours spaced at $\pi / 2$. The velocity fields $(a) u^{s}$ and $(b) w^{s}$ are shown as thin contours spaced at $1 / 4$ of the maximum disturbances, with negative values dashed. Two triangles indicate the location of the topographic peaks along the $\zeta=0$ axis.

As a result of the coordinate change, additional non-constant coefficients are introduced into the eigenvalue problem (2.15) via the derivative relations

$$
\partial_{x}=\partial_{\xi}-w^{s} \partial_{\zeta}, \quad \partial_{z}=\left(1+u^{s}\right) \partial_{\zeta} .
$$

The eigenvalue problem (2.15) is then reorganized into the matrix operator problem

$$
\left[\begin{array}{ll}
\mathbf{L}_{11} & \mathbf{L}_{12} \\
\mathbf{L}_{21} & \mathbf{L}_{22}
\end{array}\right]\left[\begin{array}{l}
\tilde{\psi} \\
\tilde{\theta}
\end{array}\right]=\lambda\left[\begin{array}{ll}
\mathbf{M} & 0 \\
0 & \mathbf{I}
\end{array}\right]\left[\begin{array}{l}
\tilde{\psi} \\
\tilde{\theta}
\end{array}\right],
$$

where the matrix entries are the linear operators ( $I$ is the identity)

$$
\left.\begin{array}{l}
\mathbf{L}_{12}=\left(1+u^{s}\right)^{-1}\left(\partial_{\xi}-w^{s} \partial_{\zeta}\right), \\
\mathbf{M}=-\partial_{\zeta}\left(\left(1+u^{s}\right) \partial_{\zeta}\right), \\
\mathbf{L}_{11}=-\mathbf{L}_{12}+\partial_{\xi}\left(1-\left(1+u^{s}\right) \mathbf{M}\right), \\
\mathbf{L}_{21}=-\mathbf{L}_{22}=\left(1+u^{s}\right) \partial_{\xi},
\end{array}\right\}
$$

and $u^{s}(\xi, \zeta)$ and $w^{s}(\xi, \zeta)$ are in isentropic coordinates. The specific form of the $\mathbf{M}$-operator is such that the matrix operator on the right-hand side of (2.20) is selfadjoint (see Strang 1986, chap. 3). This property permits application of the Arnoldi eigenvalue search algorithm to the discretization of (2.20).

\subsubsection{The numerical discretization}

In isentropic coordinates, standard second-order centred finite-differencing methods can be used to discretize the stability problem (2.20) (Iserles 1996). On the rectangular domain

$$
-L \leqslant \xi \leqslant L, \quad 0 \leqslant \zeta \leqslant H,
$$

a discretization onto an $M_{\xi} \times M_{\zeta}$ uniform grid results in a sparse matrix eigenvalue problem with dimension $2 M_{\xi} M_{\zeta}$. 


$\begin{array}{ccccccc}\text { Parameter } & L & H & M_{\xi} & M_{\zeta} & z_{D} & x_{D} \\ \text { Value } & 32 & 20 \pi & 384 & 479 & 12 \pi & 20\end{array}$

TABLE 2. Numerical parameters for the discretization (2.22) and damping layer (2.23) used for most of the eigenvalue computations in this paper.

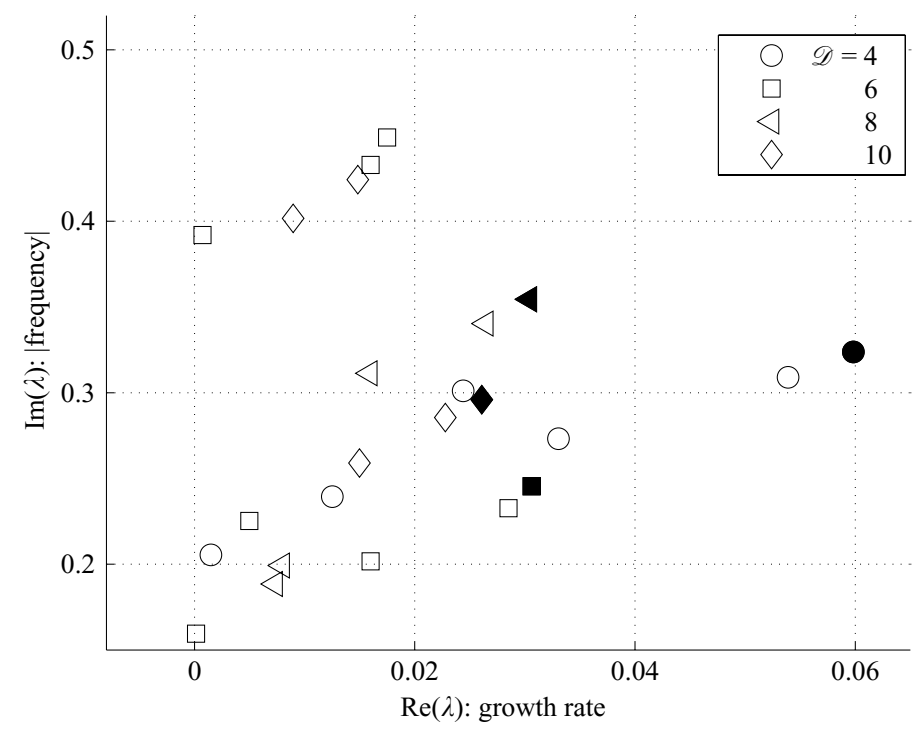

FIGURE 3. Scatterplot in the complex plane of the numerically computed unstable eigenvalues for topography of moderate height $(\mathscr{A}=0.5)$ for several values of peak separation: $\mathscr{D}=4$ (six circles), $\mathscr{D}=6$ (eight squares), $\mathscr{D}=8$ (five triangles), and $\mathscr{D}=10$ (five diamonds). The eigenvalue of the most unstable mode for each value of $\mathscr{D}$ is indicated by a filled symbol. The eigenmode corresponding to the most unstable $\mathscr{D}=4$ mode (filled circle) is shown in figure 4 Some of the scatter is explained by the analysis leading to figures 11 and 12 .

The far-field radiation conditions are imposed through the introduction of damping layers in the regions near the top and lateral boundaries

$$
x_{D} \leqslant|x| \leqslant L, \quad z_{D} \leqslant z \leqslant H .
$$

Appendices $\mathrm{A}$ and $\mathrm{B}$ elaborate on the implementation of the damping layers, and most importantly, the extrapolation of the eigenvalues to their limit for zero-damping. The numerical parameters used for the eigenvalue computations in this paper are summarized in table 2 (with the exception of the third curve in figure 12).

\section{The spatial structure of unstable modes}

\subsection{Existence of instabilities}

We begin by considering the stability for flow past twin-peak topography (2.4) of moderate height $(\mathscr{A}=0.5)$. For the flow shown in figure 1 with a peak separation of $\mathscr{D}=4$, numerical computation of the eigenvalue problem (2.20) identifies six complex-conjugate pairs of unstable eigenvalues. The six eigenvalues with positive imaginary part are indicated by circles in the scatterplot of figure 3 . The relatively slow growth and the oscillatory nature of the instabilities implied by these eigenvalues 

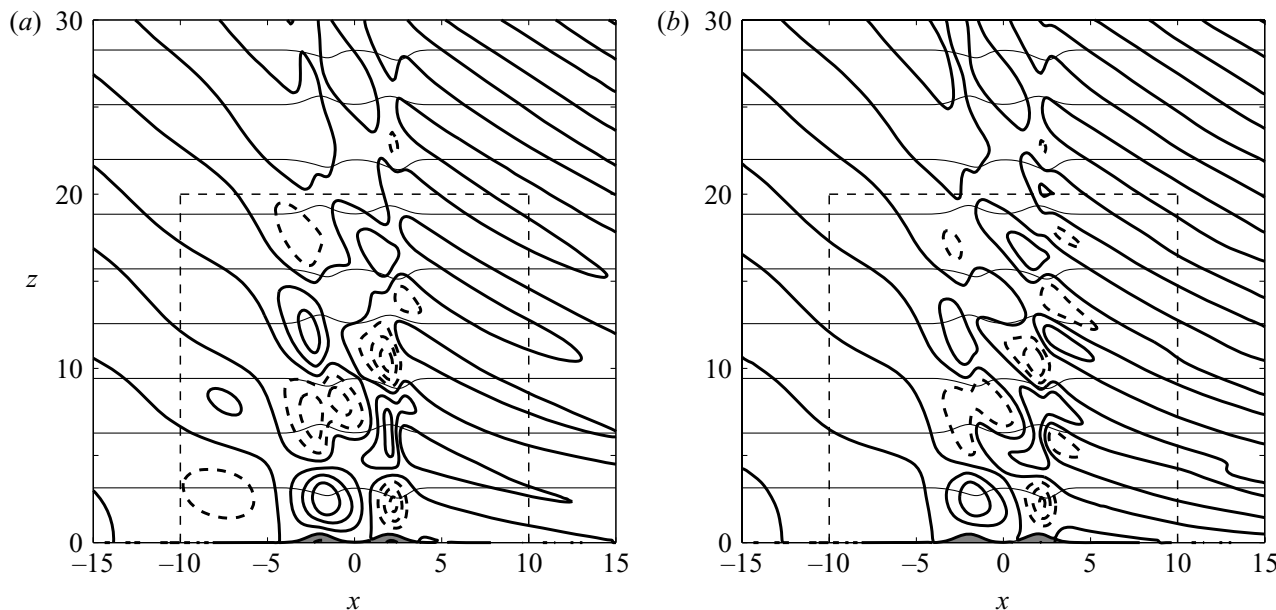

FIGURE 4. Spatial structure of the most unstable eigenmode for the flow of figure 1 $(\mathscr{A}=0.5, \mathscr{D}=4)$. The real parts of $(a) \tilde{\psi}_{4}(x, z)$ and $(b) \tilde{\theta}_{4}(x, z)$ are shown as thick contours spaced at $1 / 4$, with negative values dashed. The streamlines of the underlying steady flow are given by thin contours spaced at $\pi$. The spatial domain is larger than that used for figure 1 , whose extent is indicated by the dashed region. Furthermore, the undamped computational domain (table 2) extends beyond the plot shown here.

are consistent with direct simulations (Epifanio \& Muraki, work in progress). For larger values of peak separation $(\mathscr{D}=6,8,10)$, the computation also identifies several unstable eigenvalues. These are included in the scatterplot of figure 3, where all of the eigenvalues lie somewhat clustered in the complex plane. The most unstable eigenvalue for each value of $\mathscr{D}$ is highlighted by a filled symbol.

The existence of these unstable eigenvalues was verified to be robust to the numerical parameters of the computation - particular attention was paid to the convergence in discretization $\left(M_{\xi}, M_{\zeta}\right)$, and to the zero-damping limit (Appendix B). We conclude that these eigenvalues indicate instabilities with a physical origin.

In the above cases, the steady streamlines are not close to overturning, which suggests an instability different from wavebreaking dynamics. To grasp the nature of these computed instabilities, we focus on the analysis of the most unstable mode for the steady flow of figure 1.

\subsection{Spatial structure of the instability}

Of the six unstable modes found for the topographic flow of figure $1(\mathscr{A}=0.5, \mathscr{D}=4)$, the eigenvalue of the most unstable mode (referenced by a subscript 4) is $\lambda_{4} \approx 0.060+0.324 \mathrm{i}$. Figure 4 illustrates the spatial structure (in physical coordinates) of this mode by the real parts of $\tilde{\psi}_{4}(x, z)$ and $\tilde{\theta}_{4}(x, z)$. The complex-valued eigenmode is normalized so that the value of $\tilde{\psi}_{4}$ with the largest magnitude is 1 . Both components of the mode essentially share the same spatial character.

The largest amplitudes of the modes are found just above the surface topography. In particular, the mode naturally decays in the vertical, even though the underlying steady waves do not. Note that the damping layer $(z>12 \pi)$ is well above the top of the plot region of figure 4 . Both the vertical and horizontal scales of the eigenmode are comparable to those of the steady flow; this stands in contrast to the small-scale instabilities observed by Klemp \& Lilly (1978). 
Directly above the topography, the mode has a cellular structure. The phase of the imaginary part of the eigenmode (not shown) is such that, with increasing time, there is a horizontal drift of the cells in the upstream direction. There is also a sharp linear feature emanating (upward) from the downstream side of the second peak.

The zero contours emphasize the weak near-planar waves in the far-field regions upstream and downstream of the vertical shaft of the steady waves. The effects of the lateral damping layers $(|x|>20)$ are not visible in the plot.

The spatial features described above for the most unstable mode (figure 4) are also characteristic of the other five unstable modes for this topography $(\mathscr{A}=0.5, \mathscr{D}=4)$. Although the spatial structure has divulged little of the nature of the instability, in the next section, the mode is revealed to be a superposition of predominantly three distinct wavepackets.

\subsection{Wavepacket decomposition}

As the eigenvalue problem (2.20) has non-constant coefficients, the Fourier modes are not natural modes of the system. Nonetheless, it proves illuminating to re-characterize the spatial structure of the unstable modes in terms of their Fourier spectrum.

Although the flow domain has an irregular geometry in the physical coordinates, it is rectangular in the isentropic coordinates. As the linear modes are required to be zero on the $\zeta=0$ axis (2.16b), a well-defined (complex) Fourier transform can be applied to an odd-extension of the eigenmodes to the domain

$$
-L \leqslant \xi \leqslant L, \quad-H \leqslant \zeta \leqslant H .
$$

With this extension, the unstable streamfunction $\tilde{\psi}(\xi, \zeta)$ is now a localized function centred on the origin, and its Fourier transform can be computed.

For the most unstable mode analysed previously (figure 4), the log-magnitude Fourier spectrum of $\tilde{\psi}_{4}(\xi, \zeta)$ is shown in figure 5(a), where $k$ and $m$ denote the horizontal and vertical wavenumbers. (Note that, in the absence of topography, the isentropic $(\xi, \zeta)$ and physical $(x, z)$ space linearized equations are identical. Hence the use of $k$ and $m$ as wavenumbers in isentropic coordinates here is not inconsistent with their use in $\S 2.2$.) The reflection symmetry with respect to $m=0$ is due to the odd-extension in $\zeta$. The Fourier spectrum for $\tilde{\theta}_{4}(\xi, \zeta)$ is qualitatively similar, and is not shown here.

The spectrum of $\tilde{\psi}_{4}(\xi, \zeta)$ is concentrated along a dispersion curve (2.9) whose frequency is given by $\omega_{-}(k, m)=-\operatorname{Im}\left(\lambda_{4}\right)$ (thick dark contour). This observation suggests that the most unstable mode is composed primarily of a specific superposition of (slow) linear gravity waves, all of which share the frequency of the unstable mode itself.

In particular, there are four significant spectral peaks - three of these are boxed in figure $5(a)$. Each peak is assigned a specific value for a central wavevector which lies on the dispersion curve (indicated by a dot). The Fourier inversion of a spectral peak (in isolation) represents a wavepacket of linear waves all close to the central wavevector. Inversions of these peaks, involving only the Fourier modes delineated by their bounding boxes, are denoted by $\tilde{\psi}_{4}^{R}(\xi, \zeta), \tilde{\psi}_{4}^{U}(\xi, \zeta)$, and $\tilde{\psi}_{4}^{D}(\xi, \zeta)$, and are shown as figures $5(b-d)$. Based on the central wavevector, the group velocity (2.11) of each wavepacket is determined; its direction is indicated in each figure by the $\boldsymbol{c}_{-}^{g}$ arrow.

First, consider the wavepacket $\tilde{\psi}_{4}^{U}(\xi, \zeta)$ obtained from the spectral box labelled U. Based on its central wavevector, $\boldsymbol{k}_{4}^{U} \approx(-0.89,-1.57)$, the direction of its group velocity (2.11) is downstream and upward (giving the initial for its assigned label). As 

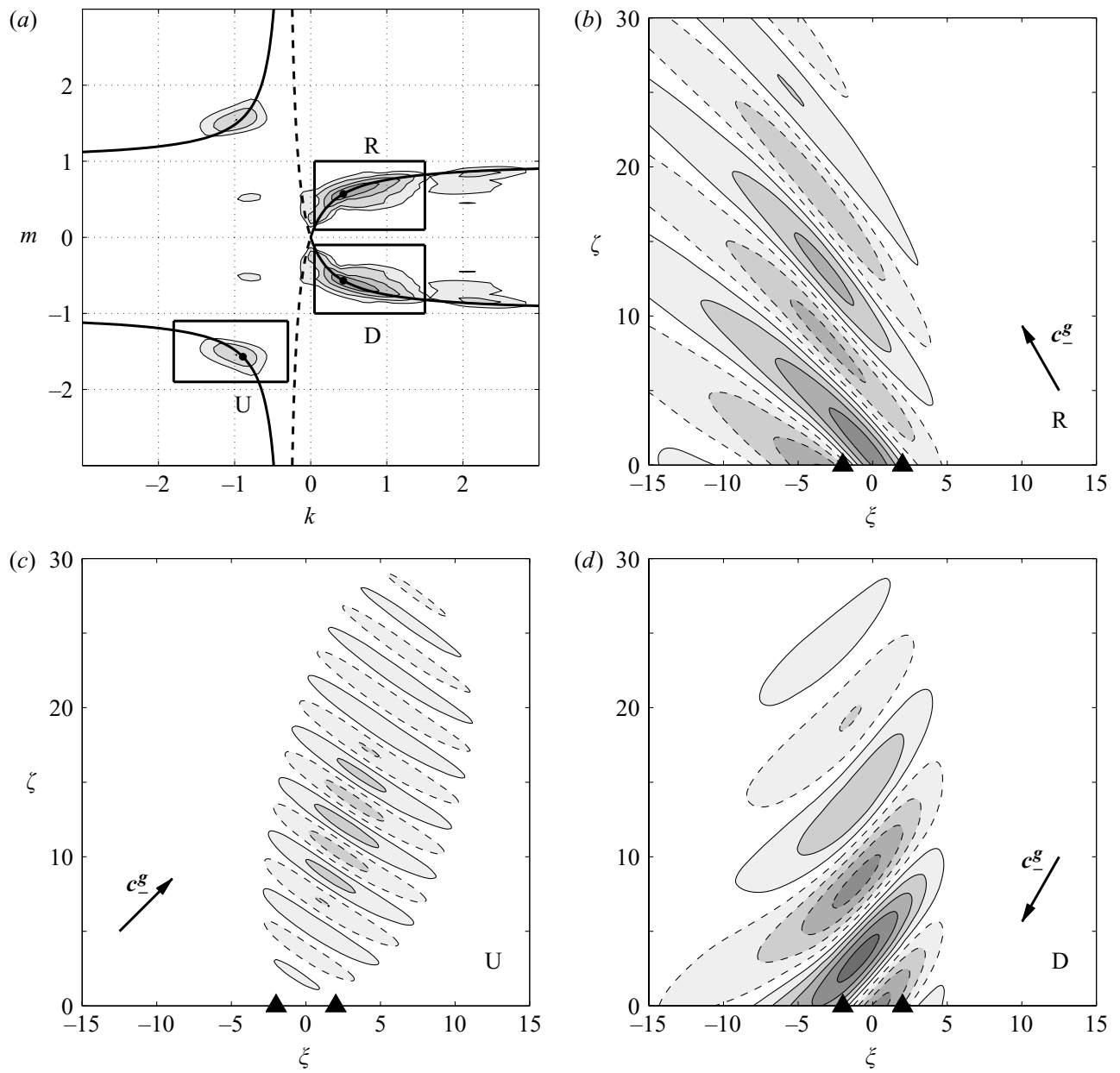

FIGURE 5. (a) Greyscale contouring of the Fourier spectrum of $\tilde{\psi}_{4}(\xi, \zeta)$. The log-magnitude of spectral amplitude is shown by thin contours spaced at $2^{-j}(j=1, \ldots, 4)$ of the maximum. Darker shading indicates larger values. The thick, solid (dashed) contours are the dispersion curves of the slow (fast) waves with frequency $\omega_{\mp}(k, m)=-\operatorname{Im}\left(\lambda_{4}\right)$. The other three panels show greyscale contouring of the real parts of the wavepackets: $(b) \tilde{\psi}_{4}^{R}(\xi, \zeta),(c) \tilde{\psi}_{4}^{U}(\xi, \zeta)$ and (d) $\tilde{\psi}_{4}^{D}(\xi, \zeta)$. Wavepackets are isolated by the boxes (R, U, D) of $(a)$. Contours are spaced at intervals of 0.1 for the range $[-0.35,0.45]$, with negative values dashed. The directions of the wavepacket group velocities $\left(\boldsymbol{c}_{-}^{g}\right)$ are indicated by the arrows.

the only wavepacket whose group velocity is directed downstream, the U-wavepacket is responsible for the near-plane waves seen downstream of the topography in figure 4 . Again by group velocity considerations, it is reasonable that the U-wavepacket exists only downstream of the first peak. Oddly, it does not extend to the ground surface, and hence, seems not to be generated directly by the topography. Lastly, the image of the spectral U-peak in the upper left quadrant has a Fourier inverse which resides entirely below the $\zeta=0$ ground plane, and is thus an artefact of the odd-extension introduced in the analysis.

The central wavevector for the D-wavepacket, $\boldsymbol{k}_{4}^{D} \approx(0.43,-0.57)$, determines a group velocity which is upstream and downward propagating. It exists upstream of a sharp cutoff at the rear edge of the topography. Consistent with its downward group 
velocity, the $\mathrm{D}$-wavepacket extends right down to the topographic surface. The phase signature on the surface is the reverse of the R-wavepacket, and identifies this third wavepacket as a surface reflected wave. The group velocity for the R-wavepacket, based on its reflection-symmetric central wavevector $\boldsymbol{k}_{4}^{R} \approx(0.43,0.57)$, is upstream and upward. Naturally, the R-wavepacket exists only upstream of where the D-wavepacket is found. The near-plane waves seen in the upstream far field of figure 4 are clearly from the R-wavepacket.

In terms of integrated $|\tilde{\psi}(\xi, \zeta)|^{2}$, the three wavepackets constitute about $90 \%$ of the original eigenmode. The more subtle spatial features as described in $\S 3.2$ can also be explained. The cellular structure above the topography is the interference pattern of the D-wavepacket with its surface reflection. Furthermore, as both phase velocities (2.10) of the D- and R-wavepackets are upstream, the time dynamics of such an interference pattern is upstream horizontal drift. Lastly, the sharp linear feature emanating from the downstream side of the second peak is associated with the abrupt ending of wave interference between the U-wavepacket and the D- and R-wavepackets.

Although not presented, the analogous wavepacket analysis based upon $\tilde{\theta}_{4}(\xi, \zeta)$ arrives at identical conclusions. The remaining question is then, by what mechanisms are these wavepackets generated? The R-wavepacket is clearly generated by the surface reflection of the D-wavepacket. However, the energy in the U- and D-wavepackets seem to appear from thin air. In the next section, we show that the U- and D-wavepackets form a resonant triad with the underlying steady flow. This nonlinear interaction is ultimately shown to be responsible for the instability.

\section{Resonant triads and instability}

\subsection{Identification of a resonant triad}

To complete the Fourier understanding of the eigenvalue problem (2.20), we are led to consider the Fourier spectrum of the non-constant coefficients which are specified by the steady flow. Since $u^{s}(\xi, \zeta)$ and $w^{s}(\xi, \zeta)$ are $2 \pi$-periodic in $\zeta$, their Fourier representations are series indexed over integer values of $m$. In figure 6(a), the absolute value of horizontal Fourier transforms of $u^{s}(\xi, \zeta)$ (solid) and $w^{s}(\xi, \zeta)$ (dashed) are plotted (over $k$ ) with baselines shifted vertically according to the value of $m$. As the velocities are real-valued, their spectra are point-symmetric about the origin (which is off-centred in the plot to reveal more of the upper right quadrant). Note that the transform in physical coordinates would only contain modes with $|m|=1$ (due to steadiness); thus, the other $m$-harmonics evident in figure 6(a) are solely due to the change of coordinates. The $m=1$ spectral peaks of the steady solution are roughly correlated with those of the Fourier transform of the topography $h(x)$

$$
\hat{h}(k)=\frac{1}{\sqrt{\pi}} \exp \left(\frac{-k^{2}}{4}\right) \cos \frac{\mathscr{D} k}{2},
$$

and imply an approximate spacing given by the formula $\Delta k \approx 2 \pi / \mathscr{D}$. The discontinuities in the spectra at $k=0$ are expected features of the steady solution (Muraki 2005).

A steady wavevector $\boldsymbol{k}_{4}^{s}$ can be identified with the largest peak of the steady $w^{s}$ spectrum (figure $6 a$ ). As demonstrated in figure 6(b), the particular choice of steady wavevector $\boldsymbol{k}_{4}^{S} \approx(1.32,1.00)$ corresponds exactly to the vector joining the $\mathrm{U}$ - and 
(a)

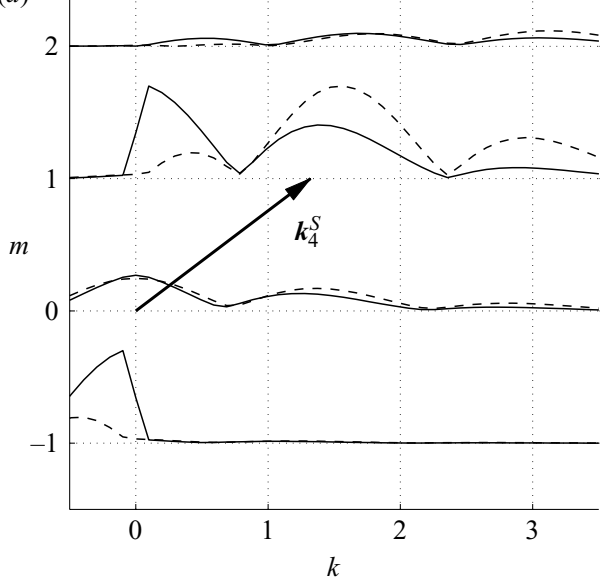

(b)

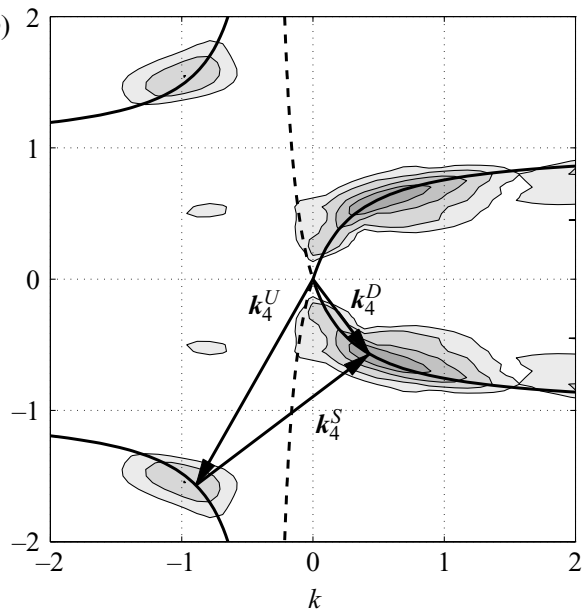

FIGURE 6. (a) Fourier spectra of $u^{s}(\xi, \zeta)$ (solid) and $w^{s}(\xi, \zeta)$ (dashed) for the steady flow of figure 2. The magnitude of the amplitudes plotted (over $k$ ) have baselines shifted vertically by their Fourier index $m$. The wavevector $\boldsymbol{k}_{4}^{s}$ defines a central wavevector for the steady flow. (b) Resonant wavevectors, $\boldsymbol{k}_{4}^{U}, \boldsymbol{k}_{4}^{D}$ and $\boldsymbol{k}_{4}^{s}$, superimposed on the spectrum of figure $5(a)$.

D-spectral peaks. This conspiracy of geometry

$$
\boldsymbol{k}^{U}+\boldsymbol{k}^{S}=\boldsymbol{k}^{D}
$$

is the spatial condition for a triad interaction. As the central wavevectors $\boldsymbol{k}_{4}^{U}$ and $\boldsymbol{k}_{4}^{D}$ were both conveniently chosen to lie along the dispersion curve $\omega_{-}(\boldsymbol{k})=-\operatorname{Im}\left(\lambda_{4}\right)$, the frequencies also satisfy the temporal resonant condition

$$
\omega_{-}\left(\boldsymbol{k}^{U}\right)+\omega_{-}\left(\boldsymbol{k}^{S}\right)=\omega_{-}\left(\boldsymbol{k}^{D}\right),
$$

since the frequency of the steady wavevector is zero. This establishes that the most unstable mode (figure 4) and the steady flow (2.14) are intrinsically connected by having spectral peaks which are close to an exact triad resonance (e.g. Craik 1985).

\subsection{A mechanism for instability}

The mathematical significance of the triad resonance lies in the fact that multiplication of Fourier modes corresponds to addition of their exponents. The triad relations (4.2), (4.3) imply that when a U-wavepacket is multiplied, in equations (2.20), by the steady flow, part of the product is a wave with the Fourier characteristics of a D-wavepacket. Because the steady flow is real-valued, it also contains the complex conjugate Fourier mode with wavevector $-\boldsymbol{k}_{4}^{s}$. This in turn implies that when a D-wavepacket is multiplied by the steady flow, part of the product is a wave with the Fourier characteristics of a U-wavepacket. Thus, the triad resonance is a signature indicating that the time dynamics of the U- and D-wavepackets are now coupled via the presence of the steady flow.

An explicit physical picture of the topographic resonance begins by considering the implications of a U-wave perturbation introduced into the steady flow above the first peak. By a group velocity argument, this perturbation U-wavepacket will propagate downstream and upward. Along the way, it will excite, via its triad interaction with the steady flow, some D-wave activity. Likewise, this new D-wavepacket disturbance, propagating upstream and downward will produce additional U-wave activity. Because the group velocities of the $\mathrm{U}$ - and D-wavepackets are counter-propagating, 
this process can be sustained in a fixed region directly over the topography. If this feedback scenario just happens to be in proper phase, the result can be a constructive resonance to exponential instability. In plasma physics and optics, this nonlinear process is known as parametric amplification (Craik 1985).

The R-wavepacket is not included in the above feedback scenario. It represents a major loss of wave energy away from the feedback process, and probably, is a significant factor in the relatively weak growth of the instability.

The unstable mode analysed in $\S 3.3$ is demonstrative proof that a positive feedback between the U- and D-wavepackets can be realized. In the next section, we demonstrate that an interaction via triads, at the level of wavepackets, is sufficient to predict the instability.

\subsection{A triad prediction for instability}

We approximate the spatial modes, $\tilde{\psi}_{4}(\xi, \zeta)$ and $\tilde{\theta}_{4}(\xi, \zeta)$, as a linear combination involving only the $\mathrm{U}$ - and $\mathrm{D}+\mathrm{R}$-wavepackets:

$$
\left.\begin{array}{l}
\tilde{\psi}_{4} \approx \alpha_{11} \tilde{\psi}_{4}^{U}+\alpha_{12}\left(\tilde{\psi}_{4}^{D}+\tilde{\psi}_{4}^{R}\right), \\
\tilde{\theta}_{4} \approx \alpha_{21} \tilde{\theta}_{4}^{U}+\alpha_{22}\left(\tilde{\theta}_{4}^{D}+\tilde{\theta}_{4}^{R}\right),
\end{array}\right\}
$$

where $\tilde{\theta}_{4}^{U}, \tilde{\theta}_{4}^{D}$ and $\tilde{\theta}_{4}^{R}$ are analogously defined for $\tilde{\theta}_{4}$. The amplitudes $\alpha_{p q}$ with $p, q=1,2$ are to be determined via an eigenvalue problem. Note that the D- and R-wavepackets are locked together because the latter is a causal reflection of the former, and their sum is required to satisfy (roughly) the zero boundary condition at the topographic surface $(2.16 b)$. The steady velocities, $u^{s}$ and $w^{s}$, are approximated by the sum of their $m= \pm 1$ harmonics.

To reduce the equations to the interaction by triads only, spatial derivatives are further approximated by spectral derivatives based only on the triad wavevectors. For example, the $\xi$-derivatives of $\tilde{\psi}_{4}^{U}, \tilde{\psi}_{4}^{D}+\tilde{\psi}_{4}^{R}$ and $u^{S}$ are approximated by $\mathrm{i} k_{4}^{U} \tilde{\psi}_{4}^{U}$, $\mathrm{i} k_{4}^{D} \tilde{\psi}_{4}^{D}+\mathrm{i} k_{4}^{R} \tilde{\psi}_{4}^{R}$ and $\mathrm{i} k_{4}^{S} u^{S}$. Projecting the action of each operator of (2.21) into a linear combination of $\mathrm{U}$ - and $\mathrm{D}+\mathrm{R}$-wavepackets (keeping only the resonant terms) reduces the eigenvalue problem (2.20) to a $4 \times 4$ system whose eigenvectors give the $\alpha_{p q}$ in (4.4).

This drastic approximation, which treats the wavepackets as envelopes of plane waves with central wavevectors of the triad, predicts instability with an eigenvalue of $0.065+0.319 \mathrm{i}$. This is within $2 \%$ of the computed eigenvalue $\lambda_{4} \approx 0.060+0.324$, and gives a quantitative demonstration for the centrality of the triad resonance as the mechanism for instability.

\subsection{A second illustration of the triad instability}

In this section, the wavepacket analysis is repeated for the most unstable eigenmode for topography with $\mathscr{A}=0.5$ and $\mathscr{D}=8$. The results for this case with twice the peak separation are completely consistent with the $\mathscr{D}=4$ example, and further support our explanation of the instability mechanism.

The five computed unstable eigenmodes for $\mathscr{D}=8$ are indicated by triangles in figure 3. The eigenvalue for the most unstable mode (denoted by subscript 8 ) is $\lambda_{8} \approx 0.031+0.355 \mathrm{i}$, and is less unstable than the $\mathscr{D}=4$ topography. Figure 7 illustrates the spatial structure by the real parts of $\tilde{\psi}_{8}(x, z)$ and $\tilde{\theta}_{8}(x, z)$. As compared to figure 4 , the basic features of the spatial structure are the same. Although, as might be expected, the lateral extent of the mode is wider, and the decay in the vertical somewhat slower. Otherwise, the most significant difference is the appearance of an extra column of cells between the peaks. 

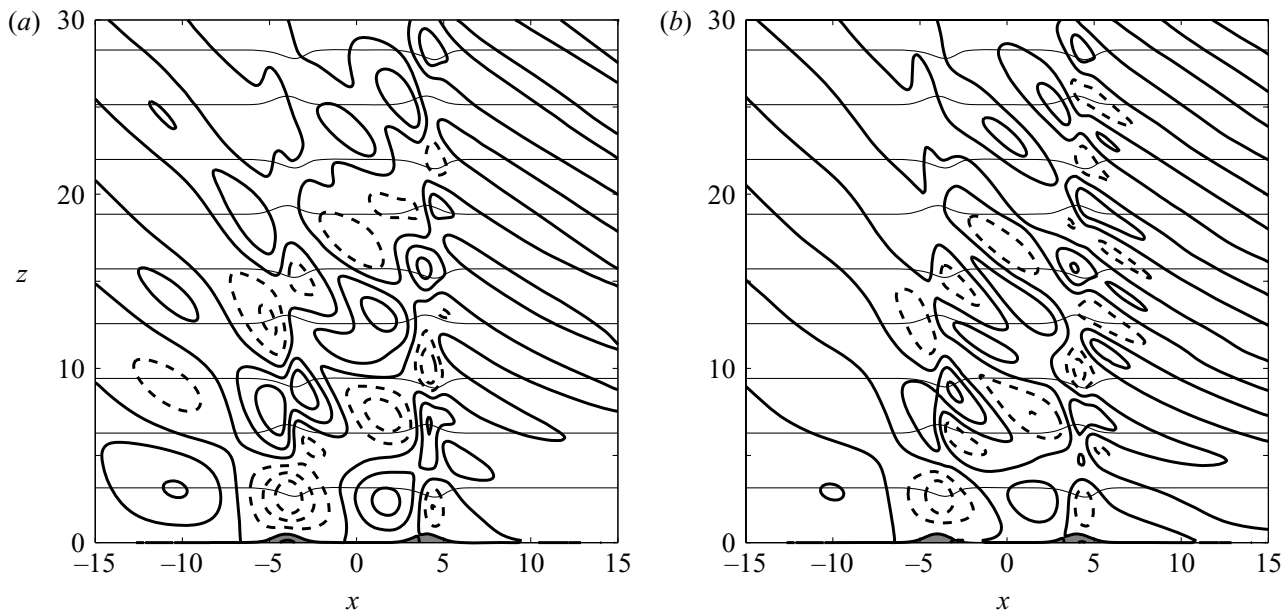

FIGURE 7. Spatial structure of the most unstable eigenmode for topography with twice the peak separation as shown in figure $4(\mathscr{A}=0.5, \mathscr{D}=8)$. The real parts of $(a) \tilde{\psi}_{8}(x, z)$ and (b) $\tilde{\theta}_{8}(x, z)$ are shown. Otherwise, plot parameters are the same as in figure 4.

Figure 8 shows the results of the wavepacket decomposition. The Fourier amplitudes are concentrated similarly along the dispersion curve $\omega_{-}(\boldsymbol{k})=-\operatorname{Im}\left(\lambda_{8}\right)$. The spatial characteristics of the wavepackets are identical to those of figure 5 , and the central wavevectors are assigned as: $\boldsymbol{k}_{8}^{U} \approx(-1.02,-1.53), \boldsymbol{k}_{8}^{D} \approx(0.40,-0.53)$, and $\boldsymbol{k}_{8}^{R} \approx(0.40,0.53)$.

The most interesting difference for this broader twin-peak case occurs in the identification of the resonant triad. As is clear from figure 9, the resonant central wavevector $\boldsymbol{k}_{8}^{S} \approx(1.42,1.00)$ for the steady flow is again the largest, but really the second significant peak of the $w^{s}$-spectrum. The triad prediction following the approximations of $\S 4.3$ gives an unstable eigenvalue of $0.036+0.356 \mathrm{i}$ which agrees well with the originally computed eigenvalue $\lambda_{8}=0.031+0.355 \mathrm{i}$.

This second example re-affirms that triad resonance is the mechanism behind this instability of steady topographic flow. It is curious that the most unstable mode for $\mathscr{D}=8$ involves a resonance with the second, steady spectral peak, and has a spatial structure which contains an extra column of cells. Resolution of these minor conundrums is addressed by a parameter study over peak separation $(\mathscr{D})$ in $\S 6$. More important however, is the question of the topographic height $(\mathscr{A})$ required for the onset of this resonant instability.

\section{A finite-height threshold for instability}

All of the unstable eigenvalues shown in the scatterplot of figure 3 are based upon a twin-peak topography with height $\mathscr{A}=0.5$. In this section, we demonstrate the existence of threshold heights at which the resonant instability first appears. For two values of peak separation $\mathscr{D}=4$ and 8 , unstable eigenvalues beginning from $\lambda_{4}$ and $\lambda_{8}$ are computed by continuation, where $\mathscr{A}$ is varied by steps of 0.01 . The most unstable growth rates are shown in figure 10. Estimated heights for the onset of resonant instabilities are approximately $\mathscr{A}=0.37$ for $\mathscr{D}=4$ and $\mathscr{A}=0.40$ for $\mathscr{D}=8$. We thus find that the height required for the onset of resonant instability is roughly only half of the height required for critical streamline overturning (approximately 0.77 

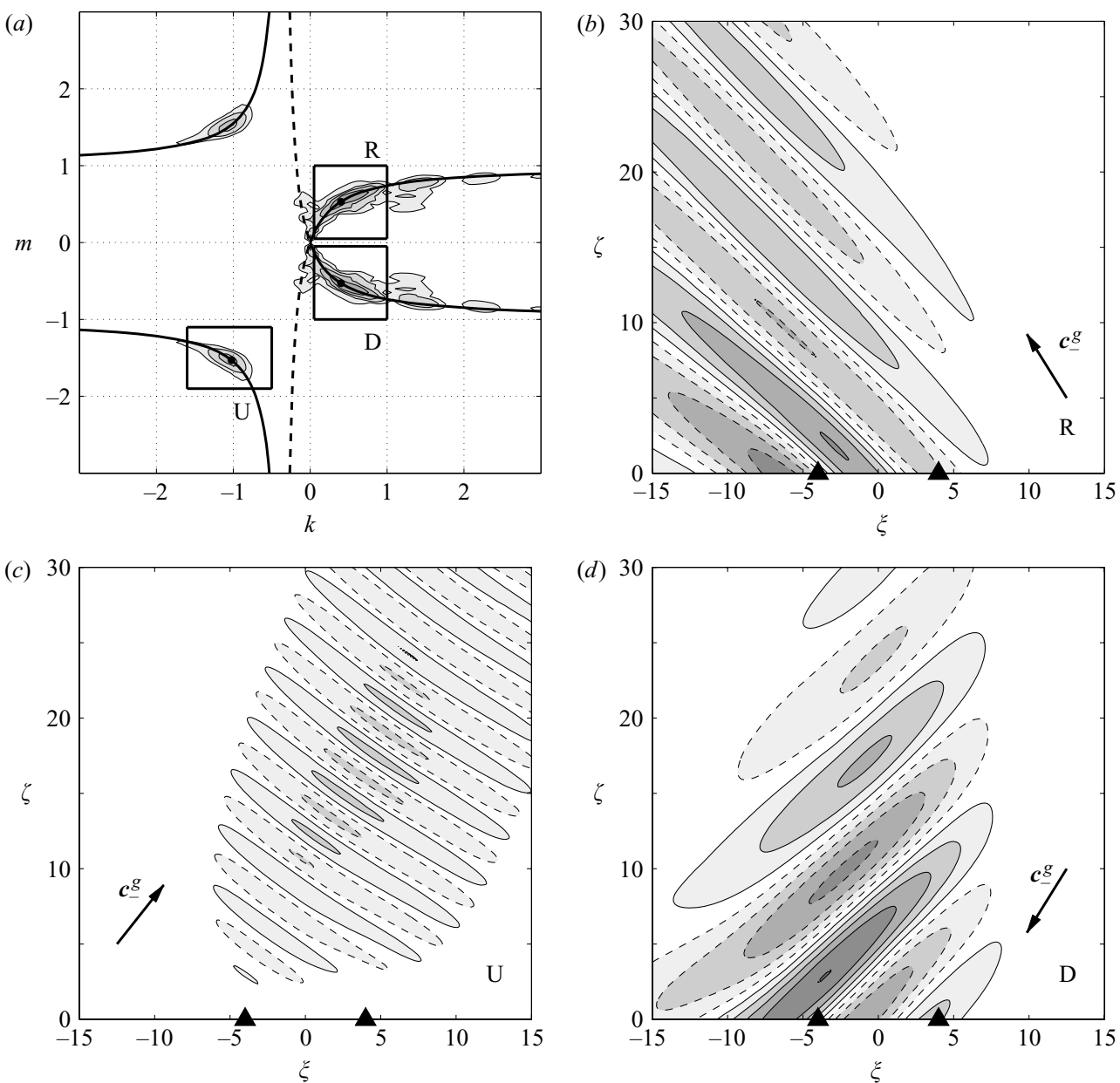

FIGURE 8. (a) Greyscale contouring of the Fourier spectrum of $\tilde{\psi}_{8}(\xi, \zeta)$. The other three panels show greyscale contouring of the real parts of the wavepackets: $(b) \widetilde{\psi}_{8}^{R}(\xi, \zeta),(c) \tilde{\psi}_{8}^{U}(\xi, \zeta)$ and (d) $\tilde{\psi}_{8}^{D}(\xi, \zeta)$. Otherwise, plot parameters are as in figure 5 .

for $\mathscr{D}=4$, and 0.79 for $\mathscr{D}=8$ ). Growth rates for both values of $\mathscr{D}$ increase with $\mathscr{A}$. Beyond $\mathscr{A}=0.55$ however, the computations are limited by spatial resolution with increasingly steep gradients in the coefficients $u^{s}(\xi, \zeta)$ and $w^{s}(\xi, \zeta)$.

Because of this computational limit in obstacle height, we are unable to access the height values near the stability threshold for single-peaked topography. This threshold is known to be in the vicinity of the height for critical streamline overturning (Laprise \& Peltier 1989a; Pierrehumbert \& Bacmeister 1987), or possibly slightly lower (Prasad, Ramirez \& Akylas 1996). Nonetheless, for a single peak at moderate heights $(\mathscr{A} \approx 0.5)$, no unstable eigenmodes were detected by our linear stability calculations.

\section{Resonant modes over topography}

\subsection{Multiple branches of instability}

As noted in $\$ 4.4$, the only changes in the properties of the most unstable mode for $\mathscr{D}=8$ was the involvement of the second spectral peak for the steady wavevector 

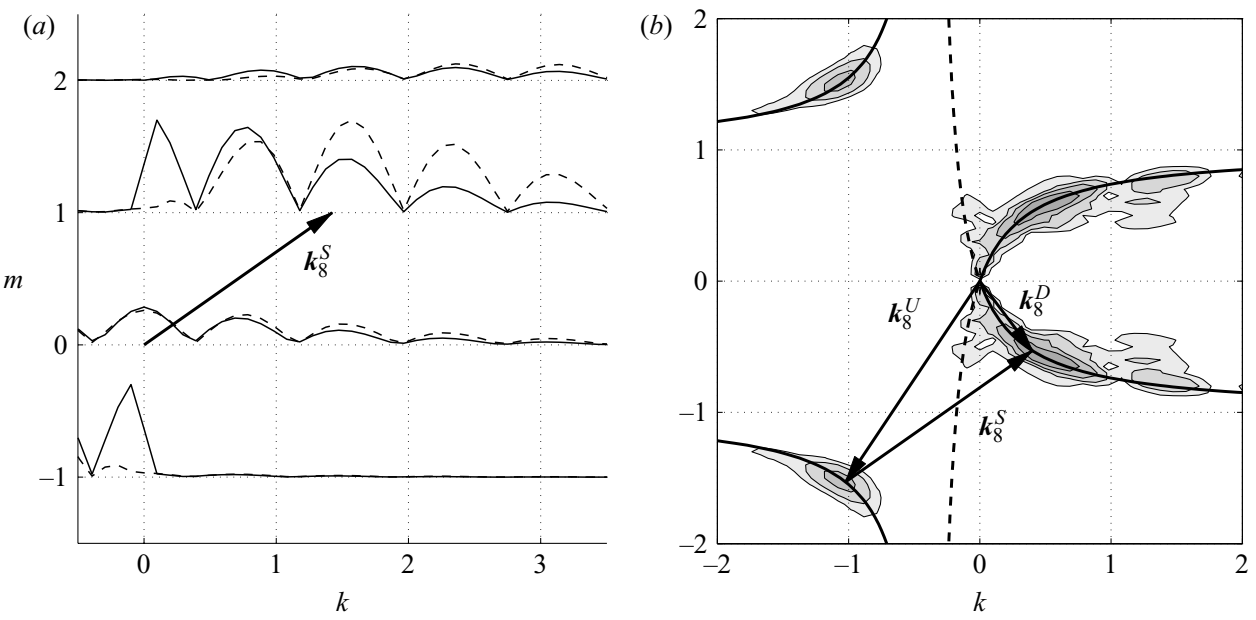

FiguRE 9. (a) Fourier spectra of $u^{s}$ (solid) and $w^{s}$ (dashed) for the steady flow with $\mathscr{D}=8$ and $\mathscr{A}=0.5$. The plot is otherwise similar to figure $6(a)$. (b) Resonant wavevectors, $\boldsymbol{k}_{8}^{U}, \boldsymbol{k}_{8}^{D}$ and $\boldsymbol{k}_{8}^{S}$, superimposed on the spectrum of figure $8(a)$.

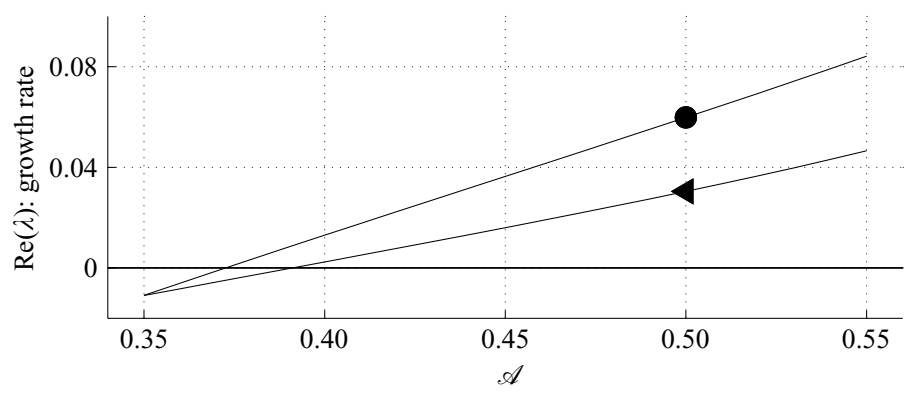

FIGURE 10. Demonstration of the finite-height threshold for the resonant instability. Curves show the growth rate of the most unstable eigenmode as a function of height $\mathscr{A}$, for two values of peak separation $\mathscr{D}=4$ and 8 . Markers indicate $\lambda_{4}$ (filled circle) and $\lambda_{8}$ (filled triangle).

$\boldsymbol{k}_{8}^{s}$ (figure $9 a$ ), and the extra column of cells above the topography (figure 7). The root of these differences is actually that $\lambda_{4}$ and $\lambda_{8}$ belong to different branches of unstable eigenvalues. The existence of distinct branches of instability is established by examining the dependence of the most unstable mode on the peak separation parameter $\mathscr{D}$.

Curves of eigenvalues as a function of peak separation (with fixed $\mathscr{A}=0.5$ ) are computed by continuation, where $\mathscr{D}$ is varied by steps of 0.1 . Beginning from $\mathscr{D}=4$ and the eigenvalue $\lambda_{4}$, a continuous branch of eigenvalues is found for the range $2 \leqslant \mathscr{D} \leqslant 9$. This curve, which includes the value $\lambda_{4}$ (filled circle), is shown in figure 11 . However, a completely distinct second branch is obtained when beginning from $\mathscr{D}=8$ and $\lambda_{8}$ (filled triangle), and spans the range $5 \leqslant \mathscr{D} \leqslant 17$. Of the instabilities shown in the scatterplot of figure 3 , the first branch also includes the most unstable eigenvalue for $\mathscr{D}=6$ (filled square), and an eigenvalue for $\mathscr{D}=8$ (open triangle). The second branch incorporates eigenvalues with $\mathscr{D}=6$ (open square) and $\mathscr{D}=10$ (filled diamond). The classification of the unstable eigenvalues around these two branches explains some of the scatter in the data of figure 3. 


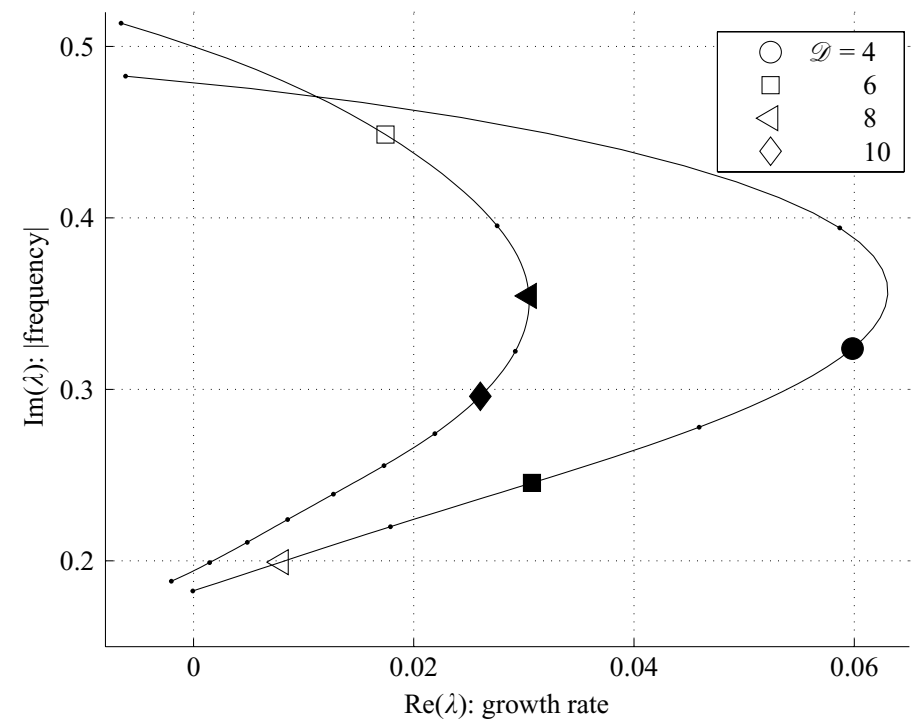

FIGURE 11. Two branches of eigenvalues as a function of peak separation $(\mathscr{D})$ for $\mathscr{A}=0.5$. The continuations were started from the eigenvalues $\lambda_{4}$ (filled circle) and $\lambda_{8}$ (filled triangle). Dots and markers indicate integer values of $\mathscr{D}$, with markers identifying eigenvalues common with figure 3 . On both branches, the frequency decreases with increasing $\mathscr{D}$.

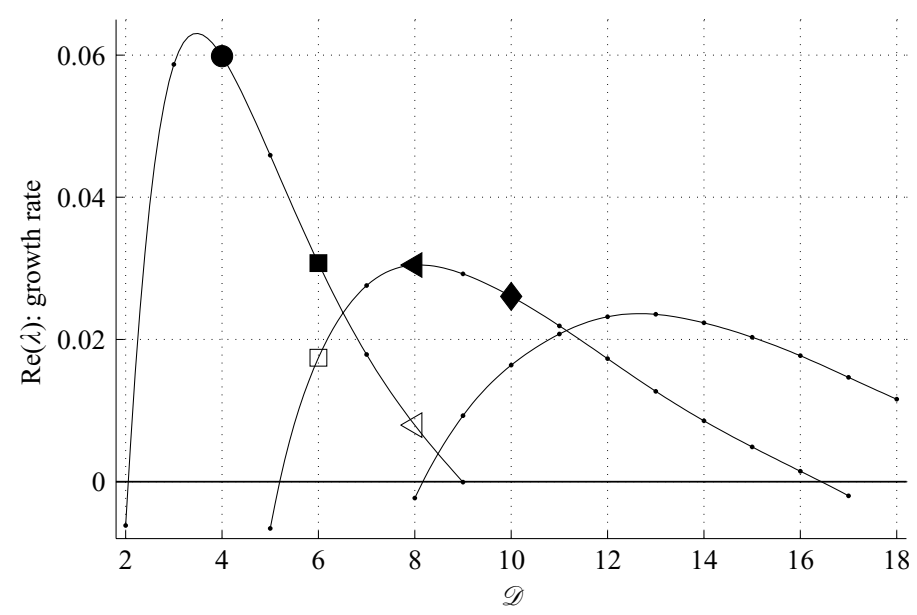

FIGURE 12. Growth rate for the first three branches of eigenvalues as a function of peak separation. The most unstable eigenvalue switches branches as $\mathscr{D}$ changes. Markers identify eigenvalues common with figure 3 .

Examination of the spatial structure for modes reveals that all modes of the second branch have the feature of the extra column of cells, while those of the first branch do not. Moreover, the second branch instabilities are always distinguished through the involvement of the second spectral peak as the steady wavevector in its resonant triad.

\subsection{Branches as resonant modes}

Growth rates for the two branches of figure 11 are replotted to show their dependence $\mathscr{D}$ in figure 12 . The filled markers clearly show that the most unstable modes for 
$\mathscr{D}=4,6$ lie on a different branch from those of $\mathscr{D}=8,10$. A switching of most unstable branch occurs for a peak separation of $\mathscr{D} \approx 6.5$. A third branch, computed at larger peak separations (also for $\mathscr{A}=0.5$ ), indicates that a subsequent switching occurs at $\mathscr{D} \approx 11.2$. This plot showing multiple local maxima of growth rate are reminiscent of the resonance curves which describe the gain characteristics of a resonant cavity.

Generically, a resonant cavity is a space containing counter-propagating waves which are coupled by a feedback interaction. In $\S 4.2$, the counter-propagation (by group velocity) of the U- and D-wavepackets was seen to be a crucial element in the physical mechanism of the instability. The cavity geometry directly affects the phase of the interaction. Maximal constructive feedback occurs for particular cavity sizes and wavelengths, which are determined by an optimal matching of phases. This is the phenomenon of resonance (Lighthill 1978).

For the topographic instability, the feedback interaction is provided by parametric amplification with the steady flow. Accordingly, for increasing peak separation, the spatial structures that resonate most strongly would be those which most naturally fit above the topography, and thus involve more wavelengths (horizontal cells). The three branches are identified by having a mode structure with $1,1.5$ and 2 wavelengths over the span of the topography. The resonant nature of the instability is clearly evident in figure 12 , where the branches characterized by fewer cells are unstable at smaller values of $\mathscr{D}$. However, what exactly determines the values of maximal growth remains a mystery. These maxima appear to be nearly equally spaced. Whether or not this indicates a resonance length (approximately 4.5) is also undecided. One intriguing fact is that the frequency associated with the three maxima are all nearly equal with $\omega \approx 0.35$.

\subsection{Stabilization of mode branches}

In the limit of small peak separation, the twin-peak topography merges into a single obstacle. However with decreasing $\mathscr{D}$, all of the resonant modes for amplitude $\mathscr{A}=0.5$ stabilize prior to the appearance of overturning in the steady flow. This suggests that the overturning instability is unrelated to the resonant instabilities discussed here. Consistent with the resonant cavity analogy, these stabilizations occur at larger values of $\mathscr{D}$ for the higher eigenvalue branches (figure 12).

In the opposite limit of large peak separation, one might anticipate that the flow becomes stable through the mutual isolation of each peak. Figure 12 again confirms that this is the case for the first two branches of instability. However, by the resonant cavity reasoning, if instabilities exist for larger values of $\mathscr{D}$, they are likely to be associated with higher branches of eigenvalues (i.e. with more wavelengths spanning the topography). Computations for very large values of $\mathscr{D}$ require wider and higher domains (to fit the topography and to encompass the wavepacket interaction region) and more resolution/accuracy (since the growth rates are likely to be small). Results from less well-resolved calculations than those shown here give an indication of the stabilization of the third resonance branch, and hint at the existence of a fourth branch. Nonetheless, these computations $(\mathscr{A}=0.5)$ are insufficient to determine the asymptotic stability definitively at large peak separations.

\section{In closing}

The instabilities analysed here are essentially a wavepacket variation of the parametric instabilities of a plane gravity wave. Resonant triad wave interactions are the primary mechanism for both. Precedents for instability via triad resonances 
are found in other studies of topographic flows. For a background stratification consisting of two layers, Nance \& Durran (1998) observed resonant triad interactions behind the breaking of lee waves trapped in the lower layer. Prasad \& Akylas (2003) also reported wave trapping from a triad produced by periodic vertical fluctuations in the Brunt-Väisälä frequency. In both these studies, an extra vertical length scale gives rise to triad resonance; in our problem, it is an extra horizontal length scale introduced by the peak separation $(\mathscr{D})$.

Although both instigated by triad resonances, this topographic version is weaker than the periodic wave instability. The growth rates are slow on the advective time scale (less than 0.1); and a finite-amplitude steady wave is required for realizing the instability $(\S 5)$. Two factors are clearly that the triad interactions are spatially limited to the steady wave region above the topography, and the loss of energy through the reflected R-wavepacket (\$4.2). Thus, these instabilities may easily go unnoticed in numerical simulations. They are readily neutralized by numerical diffusion. Mayr \& Gohm (2000) have reported steady flow over similar double-peaked topography. Epifanio \& Muraki (work in progress) only clearly observe the instability for topography with three (or more) peaks.

Based on a length scale of $\mathscr{L}=3 \mathrm{~km}$ and wind speed of $\mathscr{U}=4 \mathrm{~m} \mathrm{~s}^{-1}$ (Clark $\&$ Peltier 1977), the period of a typical most unstable mode is about $4 \mathrm{~h}$, with an e-folding growth time of roughly three and a half hours. (The period and e-folding time are calculated from the most unstable eigenvalue $\lambda_{4}$ in figure 3 . From our computations, all unstable eigenvalues yield similar hourly periods.) This hourly time scale is consistent with observed evolutions of non-stationary mountain waves (for example, Reynolds, Lamberth \& Wurtele 1968; Shutts \& Broad 1993; Ralph et al. 1997). It is clear that one source of non-stationarity can result directly from time-varying upstream conditions. Alternatively, Nance \& Durran (1998) demonstrated that temporal oscillations, of similar time scales, can result from triad resonances due to vertical variations in the Brunt-Väisälä frequency. Our resonant instability represents a closely related source of non-stationarity, born instead from horizontal variations due to the two-peak topography. It thus proposes a mechanism for mountain wave variability in the absence of wave-trapping conditions.

We have detected only large-scale unstable modes, although Klemp \& Lilly (1978) reported small-scale instabilities for their flow over periodic topography $(\$ 3.2)$. We speculate that the small-scale instabilities are associated with a different type of resonant triad, one involving the fast branch of gravity wave (2.9). In the spectral picture (say, figure $6 b$ ) this would correspond to a triad connection involving the dashed part of the dispersion curve. Such instabilities are clearly present in the (periodic domain) wave turbulence simulations of Lin (2000). We believe this type of triad does not lead to instability in our study because the group velocity of such waves are not counter-propagating. However, in a laterally periodic domain, horizontal wrap-around of wavepackets could easily sustain such a triad interaction.

We have presented the case that the topographic instability is a simple wavepacket version of a triad resonance. However, as shown in figure 13 , the spectrum of $\widetilde{\psi}(\xi, \zeta)$ for a less unstable eigenmode reveals that the underlying triad need not be unique. This example illustrates that some solutions of the non-constant coefficient stability equation (2.15) may have a richer spectrum along the dispersion curve. Nonetheless, this complication does not alter our conclusion that resonant triad interactions are ultimately responsible for the instabilities we observe.

The instabilities reported here were based on two-dimensional hydrostatic flow these assumptions were of great benefit in simplifying the analysis. However, having 


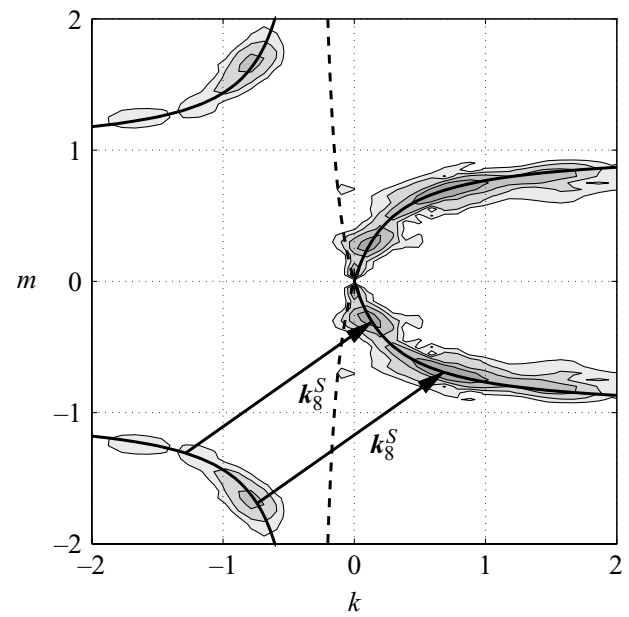

FIGURE 13. Greyscale contouring of the Fourier spectrum of $\tilde{\psi}(\xi, \zeta)$ for the third most unstable eigenvalue $(\lambda=0.016+0.311$ i) of twin-peak topography with $\mathscr{D}=8$ and $\mathscr{A}=0.5$. The spectrum suggests two possible triad connections using $\boldsymbol{k}_{8}^{s}$. Otherwise, plot features are as in figure $6(b)$.

clearly identified the triad and spatial character of the resonant instability in the hydrostatic case, provides a diagnostic basis for identifying similar instabilities in nonhydrostatic flows. In fact, non-hydrostatic flows already possess a natural horizontal scale $\mathscr{U} / \mathscr{N}$, so triad instabilities could possibly exist even for single-peak topography. Also, because the triad resonance mechanism is robust to dimensionality, it is not hard to imagine that these instabilities should at least persist in flows over (spanwise) elongated topographic obstacles. Finally, the nonlinear evolution of these instabilities to overturning and turbulence remains for future explorations.

The authors thank Craig Epifanio for his invaluable insights from numerical simulations, and for his insistence on the use of isentropic coordinates. This work was partially supported by NSERC RGPIN-238929 (Y.L., D.J.M. and D.E.A.), PIMS (Y.L.), and NSF CMG-0327658 (D.J.M).

\section{Appendix A. Numerical computation of the eigenvalues}

One subtle consequence of the change to isentropic coordinates is that the thirdorder vertical $(z)$ derivative in $(2.15)$ has no analogous term in the isentropic reformulation (2.20). This vanishing act occurs because the Jacobian is essentially a directional derivative in the $\xi$-direction. This simplification also represents a significant numerical advantage, as third derivatives at a boundary are inconvenient for secondorder centred finite-differencing. Thus, in isentropic coordinates, the second-order discretization of the stability problem (2.20) proceeds uneventfully on a uniform grid for the domain (2.22).

For the discretization, zero boundary conditions are applied at the $\zeta=0$ surface (2.18) and at the top $\zeta=H$. Laterally periodic boundary conditions are used at $\xi= \pm L$. The top and lateral conditions are artificial, and require a damping layer to remove spurious reflections and the wrap-around of waves. Details on the implementation of these damping layers are given in Appendix B.

The discretization of (2.20) is equivalent to replacing the differential operators by their corresponding finite-difference matrix operators. For an $M_{\xi} \times M_{\zeta}$ uniform 
gridding, (2.20) becomes a large generalized matrix eigenvalue problem of the form

$$
\boldsymbol{A} \boldsymbol{v}=\lambda \boldsymbol{B} \boldsymbol{v},
$$

where $\boldsymbol{A}$ and $\boldsymbol{B}$ are square sparse matrices with dimension $2 M_{\xi} M_{\zeta}$. Computations on the standard domain (table 1) use an $M_{\xi} \times M_{\zeta}=384 \times 479$ grid, and the size of the resulting matrix problem is therefore $321792^{2}$. Note that the numerical domain was increased to $H=24 \pi$ with $M_{\zeta}=575$ (and $z_{D}=16 \pi$ ) for computing the third eigenvalue branch of figure 12 , since the wave interaction region extended higher for these cases with wider topography. Finally, in several cases, the finer resolution $M_{\xi} \times M_{\zeta}=448 \times 559$ was used to verify convergence of the computed eigenvalues.

The eigenvalue problem is solved numerically using the Arnoldi iterative method as implemented in ARPACK. The solver is designed to search for a subset of the eigenvalues of large sparse matrices nearest to a given target value. This feature fits our problem nicely, as we are most interested in finding eigenvalues corresponding to unstable eigenmodes. Our searches scanned over the grid of target values defined by $0.05 j+\mathrm{i} 0.05 k$ with $j=1,2$ and $k=0$ to 20 . One restriction imposed by ARPACK is the requirement that the matrix $\boldsymbol{B}$ be symmetric and positive definite. This is true for our $\boldsymbol{B}$ matrix, as its symmetry and positive definiteness is inherited from the right-hand side operator of (2.20) (Strang 1986, chap. 3).

\section{Appendix B. Damping layers and eigenvalue extrapolation}

\section{B.1. Design of damping layers}

Following (Durran 1998), wave radiation can be effectively implemented through the introduction of Rayleigh damping terms into the horizontal momentum and thermodynamic disturbance equations (2.1)

$$
\left.\begin{array}{l}
\frac{\mathrm{D} u}{\mathrm{D} t}=-\phi_{x}-R(x, z)\left(u-u^{s}\right) \\
\frac{\mathrm{D} \theta}{\mathrm{D} t}+w=-R(x, z)\left(\theta-\theta^{s}\right) .
\end{array}\right\}
$$

The damping coefficient $R(x, z)$ is a non-negative, smooth function which is only non-zero near the boundaries of the numerical domain (2.22), and it is only used to damp disturbances from the steady solution. The damping terms then appear as modifications to the linear operators $\mathbf{L}_{11}$ and $\mathbf{L}_{22}$ in (2.21)

$$
\mathbf{L}_{11} \rightarrow \mathbf{L}_{11}+\partial_{\zeta}\left(R(\xi, \zeta)\left(1+u^{s}\right) \partial_{\zeta}\right), \quad \mathbf{L}_{22} \rightarrow \mathbf{L}_{22}+R(\xi, \zeta),
$$

where $R(\xi, \zeta)$ is in isentropic coordinates.

We define $R(x, z)$ by a top damping coefficient, $R^{T}(z)$, and a lateral damping coefficient, $R^{L}(x)$ :

$$
R(x, z)=R^{T}(z)+R^{L}(x) .
$$

The top damping coefficient $R^{T}(z)$ is given by

$$
R^{T}(z)=c_{z} \begin{cases}0 & \left(z<z_{D}\right), \\ \frac{1}{2}\left(1-\cos \left(\frac{\pi\left(z-z_{D}\right)}{H-z_{D}}\right)\right)^{2} & \left(z_{D} \leqslant z \leqslant H\right),\end{cases}
$$

which turns on at $z_{D}$ and gradually increases up to $2 c_{z}$ at $H$ (Durran 1998). The lateral damping coefficient $R^{L}(x)$, which has a form similar to (B 4), turns on at $x= \pm x_{D}$ 

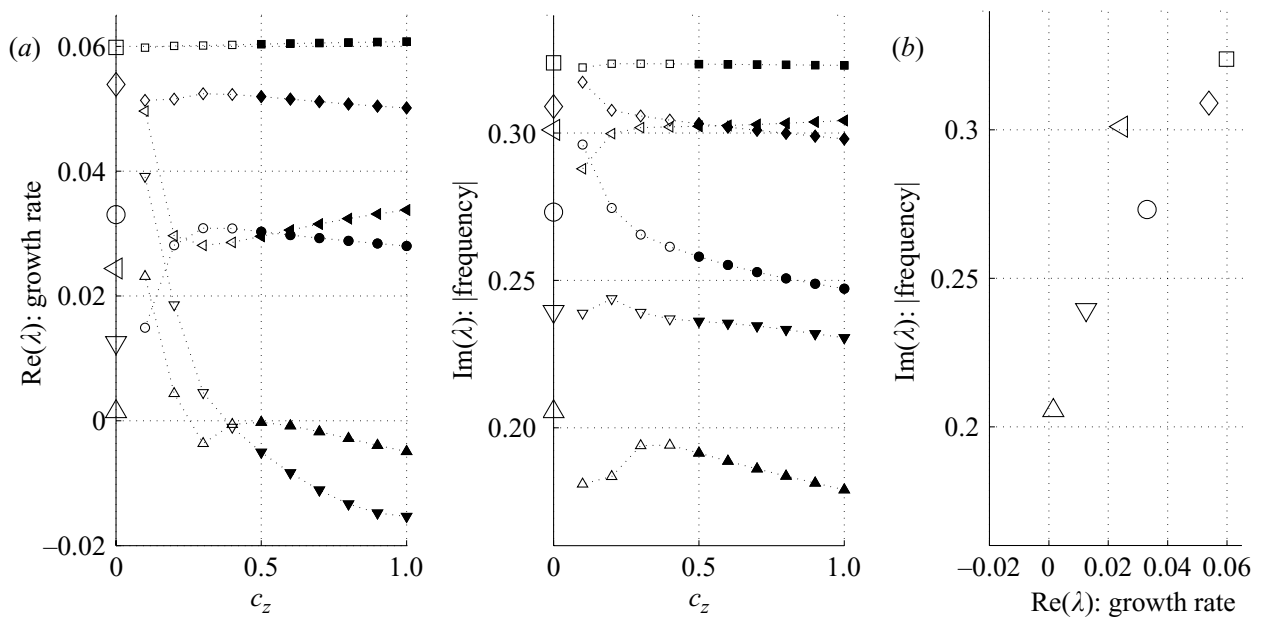

FIGURE 14. Unstable eigenvalues as a function of damping-layer strength for the topography with $\mathscr{D}=4$ and $\mathscr{A}=0.5$. (a) Like symbols identify real and imaginary parts of each eigenvalue as the coefficient $c_{z}$ is varied (B 4). Large markers at $c_{z}=0$ denote the zero damping limit obtained from linear extrapolation of the values indicated by filled markers. $(b)$ The extrapolated values shown in the complex plane.

and gradually increases up to $2 c_{x}$ at $x= \pm L$. We always set

$$
c_{x}=c_{z}\left(L-x_{D}\right) /\left(H-z_{D}\right)
$$

so that the gradient of the damping is the same for both $R^{L}(x)$ and $R^{T}(z)$.

\section{B.2. Eigenvalues in the limit of zero damping}

Recall that damping layers are introduced to the perturbation problem (2.20) to mimic the ideal situation - a semi-infinite domain over the topographic surface with an upper radiation boundary condition - by removing reflecting/wrapping waves at the numerical boundaries. While the damping layer can remove these artefacts, the resulting eigenvalues can be contaminated, and so what is really wanted are the eigenvalues in the limit of zero damping.

If the damping strength $c_{z}$ is too small, the layer will not remove all reflecting (wrapping in $x$ ) waves. On the other hand, if $c_{z}$ is too large, the damping layer can reflect waves from its gradient. For a sufficiently deep and wide damping layer, there will be an intermediate range for $c_{z}$ where the damping layer will absorb outgoing waves without reflecting anything back into the undamped region. We assume that, in this intermediate range, the eigenvalue varies linearly on $c_{z}$. (This is certainly true in the special case of constant damping coefficient $R(x, z)=R$, where each eigenvalue is simply shifted by $-R$.) Linear extrapolation is then used to obtain an estimate of the eigenvalue in the zero damping limit.

Figure 14(a) shows the six unstable eigenvalues with varying $c_{z}$ (steps of 0.025 ) for the topographic example $\mathscr{D}=4$ and $\mathscr{A}=0.5$. Real and imaginary parts of each eigenvalue are identified by distinct markers. The eigenvalues can change rapidly for $c_{z}<0.4$ - indicating that the reflected and wrapped waves at the numerical boundaries are being removed - and then relatively slowly for $c_{z}>0.4$. Estimates of the eigenvalue in the zero damping limit were obtained by linear extrapolation over the values $c_{z}>0.5$ (filled symbols). The extrapolated values at zero damping are indicated by the large symbols in figure $14(a)$ at $c_{z}=0$. These are also shown 
as the large symbols in the complex plane of figure 14(b). All eigenvalues presented in this paper are extrapolated in this manner. For most parameter values, the most unstable eigenvalue was observed to be the least sensitive to variations in the damping parameter $c_{z}$.

\section{REFERENCES}

Afanasyev, Y. \& Peltier, W. 1998 The three-dimensionalization of stratified flow over twodimensional topography. J. Atmos. Sci. 55, 19-39.

BaInes, P. 1995 Topographic Effects in Stratified Flows. Cambridge University Press.

Baines, P. \& Hoinka, K. 1985 Stratified flow over two-dimensional topography in fluid of infinite depth - a laboratory simulation. J. Atmos. Sci. 42, 1614-1630.

Clark, T. \& Peltier, W. 1977 On the evolution and stability of finite-amplitude mountain waves. J. Atmos. Sci. 34, 1715-1730.

Craik, A. 1985 Wave Interactions and Fluid Flows. Cambridge University Press.

Drazin, P. 1977 On the instability of an internal gravity wave. Proc. R. Soc. Lond. A 356, 411-432.

Durran, D. 1998 Numerical Methods for Wave Equations in Geophysical Fluid Dynamics. Springer.

EIfF, O. \& Bonneton, P. 2000 Lee-wave breaking over obstacles in stratified flow. Phys. Fluids 12, $1073-1086$.

Iserles, A. 1996 A First Course in the Numerical Analysis of Differential Equations. Cambridge University Press.

Klemp, J. \& Lilly, D. 1978 Numerical simulation of hydrostatic mountain waves. J. Atmos. Sci. 35, 78-107.

KLOSTERmeyer, J. 1982 On parametric instabilities of finite-amplitude internal gravity waves. J. Fluid Mech. 119, 367-377.

LAPrise, R. \& Peltier, W. $1989 a$ The linear stability of nonlinear mountain waves: implications for the understanding of severe downslope windstorms. J. Atmos. Sci. 46, 545-564.

Laprise, R. \& Peltier, W. $1989 b$ The structure and energetics of transient eddies in a numerical simulation of breaking mountain waves. J. Atmos. Sci. 46, 565-585.

LAPrise, R. \& Peltier, W. 1989c On the structural characteristics of steady finite-amplitude mountain waves over bell-shaped topography. J. Atmos. Sci. 46, 586-596.

Lighthill, J. 1978 Waves in Fluids. Cambridge University Press.

LiLLY, D. \& KLEMP, J. 1979 The effects of terrain shape on nonlinear hydrostatic mountain waves. J. Fluid Mech. 96, 241-261.

Lin, C. 2000 Energy transfer in a fluid with a uniform density gradient. Dyn. Atmos. Oceans 32, $113-134$.

LoNG, R. 1953 Some aspects of the flow of stratified fluids. i. a theoretical investigation. Tellus $\mathbf{5}$, $42-58$.

LoNG, R. 1955 Some aspects of the flow of stratified fluids. iii. continuous density gradients. Tellus 7, 341-357.

MaYr, G. \& Gohm, A. 2000 2d airflow over a double bell-shaped mountain. Met. Atmos. Phys. 72, $13-27$.

MIED, R. 1976 The occurrence of parametric instabilities in finite-amplitude internal gravity waves. J. Fluid Mech. 78, 763-784.

Miles, J. \& Huppert, H. 1968 Lee waves in a stratified flow. Part 2. semi-circular obstacle. J. Fluid Mech. 33, 803-814.

MuRAKI, D. 2005 The large amplitude wave solutions of Long's theory for 2D topographic flow. J. Atmos. Sci. (submitted).

Nance, L. \& Durran, D. 1998 A modeling study of nonstationary trapped mountain lee waves. Part ii: Nonlinearity. J. Atmos. Sci. 15, 1429-1445.

Phillips, O. 1968 The interaction trapping of internal gravity waves. J. Fluid Mech. 34, 407-416.

Pierrehumbert, R. \& Bacmeister, J. 1987 On the realizability of Long's model solutions for nonlinear stratified flow over an obstacle. In Proc. 3rd Intl Symp on Stratified Flows (ed. E. J. List \& G. Jirka). ASCE.

Pierrehumbert, R. \& Wyman, B. 1985 Upstream effects of mesoscale mountains. J. Atmos. Sci. 42, $977-1003$. 
Prasad, D. \& Akylas, T. 2003 Wave trapping and upstream influence in stratified flow of large depth. J. Fluid Mech. 491, 301-324.

Prasad, D., Ramirez, J. \& Akylas, T. 1996 Stability of stratified flow of large depth over finiteamplitude topography. J. Fluid Mech. 320, 369-394.

Ralph, F., Neiman, P., Keller, T., Levinson, D. \& Fedor, L. 1997 Observations, simulations, and analysis of nonstationary trapped lee waves. J. Atmos. Sci. 54, 1308-1333.

Reynolds, R., LAmberth, R. \& WURTELE, M. 1968 Investigation of a complex mountain wave situation. J. Appl. Met. 7, 353-358.

Salmon, R. 1998 Geophysical Fluid Dynamics. Oxford University Press.

Scinocca, J. \& Peltier, W. 1993 The instability of Long's stationary solution and the evolution toward severe downslope windstorm flow. Part i: Nested grid numerical simulations. J. Atmos. Sci. 50, 2245-2263.

Shutts, G. \& Broad, A. 1993 A case study of lee waves over the lake district in northern England. Q. J. R. Met. Soc. 33, 377-408.

Sмith, R. 1977 The steepening of hydrostatic mountain waves. J. Atmos. Sci. 34, 1634-1654.

Smith, R. 1979 The influence of mountains on the atmosphere. Adv. Geophys. 21, 87-230.

Staquet, C. \& Sommeria, J. 2002 Internal gravity waves: from instabilities to turbulence. Annu. Rev. Fluid Mech. 34, 559-593.

Strang, G. 1986 Introduction to Applied Mathematics. Wellesley-Cambridge Press.

Wurtele, M., Sharman, R. \& Datta, A. 1996 Atmospheric lee waves. Annu. Rev. Fluid Mech. 28, 429-476. 